\title{
ONLY A SMALL FRACTION OF CELLS PRODUCE ASSEMBLED CAPSIDS DURING TRANSFECTION-BASED MANUFACTURING OF ADENO-ASSOCIATED VIRUS VECTORS
}

\author{
Shantoshini Dash ${ }^{1}$, David Sharon ${ }^{2}$, Alaka Mullick ${ }^{3}$, and Amine Kamen ${ }^{1}$ \\ ${ }^{1}$ McGill University \\ ${ }^{2}$ McGill University Faculty of Engineering \\ ${ }^{3}$ National Research Council Canada
}

November 14, 2021

\begin{abstract}
Plasmid transfection of mammalian cells is the dominant platform used to produce adeno-associated virus (AAV) vectors for clinical and research applications. Low yields from this platform currently make it difficult to supply these activities with adequate material. In an effort to better understand the current limitations of transfection-based manufacturing, this study examines what proportion of cells in a model transfection produce appreciable amounts of assembled AAV capsid. Using conformation-specific antibody staining and flow cytometry we report the surprising result that despite obtaining high transfection efficiencies and nominal vector yields in our model system, only 5-10\% of cells appear to produce measurable levels of assembled AAV capsids. This finding implies that considerable increases in vector titer could be realized through increasing the proportion of productive cells. Furthermore, we suggest that the flow cytometry assay used here to quantify productive cells may be a useful metric for future optimization of transfection-based AAV vector manufacturing platforms.
\end{abstract}

ONLY A SMALL FRACTION OF CELLS PRODUCE ASSEMBLED CAPSIDS DURING TRANSFECTION-BASED MANUFACTURING OF ADENO-ASSOCIATED VIRUS VECTORS

S Dash $^{1 *}$, DM Sharon ${ }^{*}$, A Mullik ${ }^{2}$, AA Kamen ${ }^{1,2}$,

Author affiliations:

* These authors are equal contributors

${ }^{1}$ Department of Bioengineering, McGill University, Montreal, QC, Canada

${ }^{2}$ Human Health Therapeutics, National Research Council of Canada, Montreal, QC, Canada

Corresponding author

Corresponding author contact information:

Name: Amine A. Kamen

Email: amine.kamen@mcgill.ca

Phone: 1-514-398-5775 
Address: McGill University Department of Bioengineering

McConnell Engineering Building, Room 3633480 Rue University, H3A 2K6 Montréal, QC Canada

Funding: National Research Council of Canada, Grant number: CGT-602-1

Keywords: Adeno-associated virus, transfection, viral vectors, gene therapy

\section{ABSTRACT}

Plasmid transfection of mammalian cells is the dominant platform used to produce adeno-associated virus (AAV) vectors for clinical and research applications. Low yields from this platform currently make it difficult to supply these activities with adequate material. In an effort to better understand the current limitations of transfection-based manufacturing, this study examines what proportion of cells in a model transfection produce appreciable amounts of assembled AAV capsid. Using conformation-specific antibody staining and flow cytometry we report the surprising result that despite obtaining high transfection efficiencies and nominal vector yields in our model system, only $5-10 \%$ of cells appear to produce measurable levels of assembled AAV capsids. This finding implies that considerable increases in vector titer could be realized through increasing the proportion of productive cells. Furthermore, we suggest that the flow cytometry assay used here to quantify productive cells may be a useful metric for future optimization of transfection-based AAV vector manufacturing platforms.

\section{MAIN TEXT}

Adeno-associated viruses (AAV) have proven to be safe and efficacious gene transfer vectors, with 149 completed or ongoing clinical trials and 5 approved therapies for various forms of cell and gene therapy (Kuzmin et al., 2021). The bulk of AAV vectors used for research, clinical trials, and approved therapies are currently produced by multi-plasmid transfection of mammalian cells (Clement \& Grieger, 2016; Wang, Tai, \& Gao, 2019). Though variations on the process exist, production of AAV by transfection typically uses three plasmids: a transfer plasmid encoding the gene of interest flanked by viral inverted terminal repeat elements; a helper plasmid encoding the minimal helper virus genes necessary for the AAV lifecycle; and a packaging plasmid containing the AAV REPand CAP genes (D. Sharon \& Kamen, 2018; Wang et al., 2019).

In this study HEK-293SF cells producing an AAV2-GFP vector were used as a model to determine what proportion of transfected cells generate fully assembled capsids during vector production. Commonly used cationic transfection reagents (polyethyleneimine, calcium phosphate, lipofectamine, etc.) coprecipitate heterogeneous plasmid mixtures into larger complexes for transit across the cell membrane, and so in theory all successfully transfected cells should contain the genetic elements necessary to produce AAV (Cardarelli et al., 2016; Erbacher et al., 2004; Fus-Kujawa et al., 2021). Reported transfection efficiencies (measured by the expression of a fluorescent marker) for well optimized processes range from 40-60\% (Chahal, Schulze, Tran, Montes, \& Kamen, 2014; Nguyen et al., 2021). However, the degree to which transfection efficiency corresponds with the proportion of productive cells in the culture remains largely untested. Clarifying this will give an indication of how much of the cell biomass is being utilized with current AAV manufacturing protocols, and in turn how much those protocols might be improved.

To establish a model for subsequent experiments, a plasmid transfection to produce AAV2-GFP was carried out on HEK-293SF cells in suspension, based on an optimized process developed by Chahal et al. (Chahal et al., 2014). As seen in Figure 1a, cell density was relatively stable up to the harvest point at 48 hours posttransfection (hpt), the previously determined optimal harvest point for this process (Chahal et al., 2014). Volumetric vector yields at $48 \mathrm{hpt}$ were in the range of $10^{9} \mathrm{VG} / \mathrm{ml}$ (Figure 1b). Overall, the measurements obtained in Figure 1 are in line with previous studies where AAV vectors were produced by transfection in HEK-293 or derivative cell lines.

To determine what proportion of cells in our transfection model produce fully assembled vector capsids, transfected cells were stained with a conformation-specific antibody that binds only to assembled viral capsids, allowing the measurement of this subpopulation by flow cytometry (Wobus et al., 2000; Xiao, 
Warrington, Hearing, Hughes, \& Muzyczka, 2002). The presence of a GFP expression cassette on the transfer plasmid allowed simultaneous assessment of transfection efficiency. Cells infected with replication competent (RC) AAV2 and a human adenovirus type 5 (hAd5) helper virus were used as a positive control for capsid assembly.

As shown in Figure 2a, mean transfection efficiency at $48 \mathrm{hpt}$ was roughly $60 \%$. However, only a small fraction ( $\sim 7 \%$ ) of transfected cells produced measurable amounts of assembled AAV capsid. This contrasts with the RC AAV2 and helper virus infected control where, as expected, nearly all cells are positive for assembled AAV capsid. Note that AAV capsids will spontaneously assemble without necessarily encapsulating a viral genome, and so these measurements may be an overestimate of the proportion productive cells in the culture (Hajba \& Guttman, 2020). These results were independently replicated by separate teams at McGill University and the National Research Council of Canada

The observation that only $7 \%$ of transfected cells appeared to produce assembled AAV capsids raised the question as to what proportion of cells were expressing the necessary factors for AAV vector production. The three transfected plasmids collectively encode $~ 17$ protein and RNA elements, making measurement of every factor impractical (D. Sharon \& Kamen, 2018; Wang et al., 2019). Instead, a subset of proteins from each of the three transfected plasmids were visualized in transfected cells via immunofluorescence.

As shown in Figure 3, GFP was broadly expressed in imaged cells. Interestingly though, GFP expression does not seem to reliably indicate coexpression of AAV capsid monomers or the hAd5 E2A helper factor, both of which were expressed at detectable levels in a much smaller subset of cells. This may partially explain the observation in Figure $2 \mathrm{~b}$ that only $\sim 7 \%$ transfected cells contain assembled AAV capsids, despite an apparent transfection efficiency nearly tenfold higher.

Low yields in transfection-based AAV vector production platforms are a long-standing issue, and have spurred the development of numerous alternatives. Plasmid-free systems such as baculovirus expression vectors, herpesvirus vectors, and more recent self-silencing adenoviral systems boast significantly increased yields and are far more amenable to scaleup (Cawood, 2020; D. Sharon \& Kamen, 2018). There have also been attempts both within academia and industry to develop a stable producer cell line, though little of the latter work is published (Clement \& Grieger, 2016). While these platforms may supplant transfection for late-stage and approved therapies, the unparalleled speed and simplicity of transfection-based manufacturing means it is likely to remain a mainstay of AAV vector production for early clinical and research applications in the foreseeable future.

The results presented here indicate that current transfection-based AAV vector production protocols utilize only a fraction of the available cell biomass, and that significant increases in yield may yet be realized. To this end, the innovation and optimization of this process remains an active area of research. Computational work modeling plasmid uptake, expression, and vector assembly kinetics in HEK-293 has demonstrated utility in identifying molecular bottlenecks to improve vector yield and quality (Nguyen et al., 2021). Design of Experiments approaches to optimize process parameters have also recently been shown to be effective in increasing AAV vector yields across a wide range of serotypes (Zhao et al., 2020).

The results of Figure 2 also demonstrate that the expression of a transfection marker does not necessarily imply a cell is producing AAV vector particles. While transfection efficiency remains an important process development metric, the confirmation-specific antibody staining against assembled AAV capsids that was used here may also prove useful in the future development and optimization of transfection-based AAV vector platforms.

\section{METHODS}

Cell culture and transfection

HEK-293SF cells were maintained in serum-free suspension as previously described (D. M. Sharon et al., 2020). All transfections were performed at a cell density of $10^{6} \mathrm{cell} / \mathrm{ml}$ using linear polyethylenimine with 
a mean molecular weight of 25000Da (Polysciences) complexed with plasmid DNA at a 1:2 ratio. The final concentration of plasmid DNA in all cases was $1 \mathrm{ug} / \mathrm{ml}$.

Plasmids

For the generation of rAAV2, plasmids pAdDeltaF6 (Addgene \#112867), pAAV-RC2 (cell biolabs inc.), and pAAV-CMV-GFP (Addgene \#67634) were transfected in a 1:1:1 molar ratio. pAdDeltaF6 was a gift from James M. Wilson and pAAV-CMV-GFP was a gift from Connie Cepko (Xiong, MacColl Garfinkel, Li, Benowitz, \& Cepko, 2015). Stocks of RC AAV2 were generated by equimolar transfection of pAdDeltaF6 and pAV2-Cla. pAV2-Cla, which contains a sequence identical to wildtype AAV2 apart from a point mutation to generate a Cla1 restriction site in the 3' UTR. pAV2-Cla was generously provided by Dr. Thomas Webber.

Replication competent $(R C)$ AAV2 infection

Initial stocks of RC AAV2 were generated via plasmid transfection and titrated by dPCR. When using RC AAV2 infection as a positive control for capsid assembly, HEK-293SF cells at an initial cell density of $10^{6}$ cell $/ \mathrm{ml}$ were infected with RC AAV2 stocks and hAd5 and an MOI of 10.

Flow cytometry

Cells were fixed and permeabilized as previously described (Kerviel et al., 2016). Flow cytometry was carried out on the BD FACSJazz (BD Biosciences) and analyzed on FlowJo v10. Assembled particles of AAV2 were detected by staining with anti A20R (Progen) labelled with AlexaFluor 594 (Invitrogen).

\section{Microscopy}

HEK-293SF cells were seeded at low confluence on a 35mm plate with coverslip (MatTek). 24h post seeding, the cells were transfected for the production of AAV2-GFP. 72h post seeding, the media was removed, and the cells fixed and stained as previously described (Kerviel et al., 2016). AAV capsid monomers were detected with anti-VP1/VP2/VP1 (Progen) labelled with AlexaFluor 700 (Invitrogen), hAd5 E2A by antiE2A labelled with AlexaFluor 350 (Invitrogen). Anti-E2A was a gift from Arnold J. Levine (Reich, Sarnow, Duprey, \& Levine, 1983). Antibodies were incubated at room temperature for 1 hour. Cells were then imaged using an Olympus IX-83 confocal microscope. The images were analyzed using FIJI.

\section{$A A V$ genome quantification by digital droplet PCR}

HEK-293SF cells in culture media were freeze-thawed three times to lyse cells. Clarified lysates were then incubated with $5 \mathrm{U} / \mathrm{ml}$ Benzonase to digest unencapsulated DNA. Encapsulate genomes were then purified with the High Pure Viral DNA Extraction kit (Roche Diagnostics) and quantified on the QX200 Digital Droplet PCR system (Bio-Rad) as previously described (Furuta-Hanawa, Yamaguchi, \& Uchida, 2019).

\section{ACKNOWLEDGEMENTS}

The authors would like to acknowledge the financial support from NRC, project NRC CGT-602-1 and Fonds de recherche du Québec -TheCell (Thérapie cellulaire).

The authors declare no conflict of interest.

\section{REFERENCES}

Cardarelli, F., Digiacomo, L., Marchini, C., Amici, A., Salomone, F., Fiume, G., . . Caracciolo, G. (2016). The intracellular trafficking mechanism of Lipofectamine-based transfection reagents and its implication for gene delivery. Sci Rep, 6 , 25879. doi:10.1038/srep25879

Cawood, R. (2020). Redefining AAV Manufacturing: The Time Is Now. Retrieved from https://www.genengnews.com/magazine/redefining-aav-manufacturing-the-time-is-now/

Chahal, P. S., Schulze, E., Tran, R., Montes, J., \& Kamen, A. A. (2014). Production of adeno-associated virus (AAV) serotypes by transient transfection of HEK293 cell suspension cultures for gene delivery. $J$ Virol Methods, 196 , 163-173. doi:10.1016/j.jviromet.2013.10.038 
Clement, N., \& Grieger, J. C. (2016). Manufacturing of recombinant adeno-associated viral vectors for clinical trials. Mol Ther Methods Clin Dev, 3, 16002. doi:10.1038/mtm.2016.2

Erbacher, P., Bettinger, T., Brion, E., Coll, J. L., Plank, C., Behr, J. P., \& Remy, J. S. (2004). Genuine DNA/polyethylenimine (PEI) complexes improve transfection properties and cell survival. J Drug Target, 12 (4), 223-236. doi:10.1080/10611860410001723487

Furuta-Hanawa, B., Yamaguchi, T., \& Uchida, E. (2019). Two-Dimensional Droplet Digital PCR as a Tool for Titration and Integrity Evaluation of Recombinant Adeno-Associated Viral Vectors. Hum Gene Ther Methods, 30 (4), 127-136. doi:10.1089/hgtb.2019.031

Fus-Kujawa, A., Prus, P., Bajdak-Rusinek, K., Teper, P., Gawron, K., Kowalczuk, A., \& Sieron, A. L. (2021). An Overview of Methods and Tools for Transfection of Eukaryotic Cells in vitro. Front Bioeng Biotechnol, 9 , 701031. doi:10.3389/fbioe.2021.701031

Hajba, L., \& Guttman, A. (2020). Recent Advances in the Analysis Full/Empty Capsid Ratio and Genome Integrity of Adeno-associated Virus (AAV) Gene Delivery Vectors. Curr Mol Med, 20 (10), 806-813. doi:10.2174/1566524020999200730181042

Kerviel, A., Dash, S., Moncorge, O., Panthu, B., Prchal, J., Decimo, D., . . . Muriaux, D. (2016). Involvement of an Arginine Triplet in M1 Matrix Protein Interaction with Membranes and in M1 Recruitment into Virus-Like Particles of the Influenza A(H1N1)pdm09 Virus. PLoS One, 11 (11), e0165421. doi:10.1371/journal.pone.0165421

Kuzmin, D. A., Shutova, M. V., Johnston, N. R., Smith, O. P., Fedorin, V. V., Kukushkin, Y. S., . . . Johnstone, E. C. (2021). The clinical landscape for AAV gene therapies. Nat Rev Drug Discov, 20 (3), 173-174. doi:10.1038/d41573-021-00017-7

Nguyen, T. N. T., Sha, S., Hong, M. S., Maloney, A. J., Barone, P. W., Neufeld, C., . . Braatz, R. D. (2021). Mechanistic model for production of recombinant adeno-associated virus via triple transfection of HEK293 cells. Mol Ther Methods Clin Dev, 21 , 642-655. doi:10.1016/j.omtm.2021.04.006

Reich, N. C., Sarnow, P., Duprey, E., \& Levine, A. J. (1983). Monoclonal antibodies which recognize native and denatured forms of the adenovirus DNA-binding protein. Virology, 128 (2), 480-484. doi:10.1016/00426822(83)90274-x

Sharon, D., \& Kamen, A. (2018). Advancements in the design and scalable production of viral gene transfer vectors. Biotechnol Bioeng, 115 (1), 25-40. doi:10.1002/bit.26461

Sharon, D. M., Nesdoly, S., Yang, H. J., Gelinas, J. F., Xia, Y., Ansorge, S., \& Kamen, A. A. (2020). A pooled genome-wide screening strategy to identify and rank influenza host restriction factors in cell-based vaccine production platforms. Sci Rep, 10 (1), 12166. doi:10.1038/s41598-020-68934-y

Wang, D., Tai, P. W. L., \& Gao, G. (2019). Adeno-associated virus vector as a platform for gene therapy delivery. Nat Rev Drug Discov, 18 (5), 358-378. doi:10.1038/s41573-019-0012-9

Wobus, C. E., Hugle-Dorr, B., Girod, A., Petersen, G., Hallek, M., \& Kleinschmidt, J. A. (2000). Monoclonal antibodies against the adeno-associated virus type 2 (AAV-2) capsid: epitope mapping and identification of capsid domains involved in AAV-2-cell interaction and neutralization of AAV-2 infection. $J$ Virol, 74 (19), 9281-9293. doi:10.1128/jvi.74.19.9281-9293.2000

Xiao, W., Warrington, K. H., Jr., Hearing, P., Hughes, J., \& Muzyczka, N. (2002). Adenovirusfacilitated nuclear translocation of adeno-associated virus type 2. J Virol, 76 (22), 11505-11517. doi:10.1128/jvi.76.22.11505-11517.2002

Xiong, W., MacColl Garfinkel, A. E., Li, Y., Benowitz, L. I., \& Cepko, C. L. (2015). NRF2 promotes neuronal survival in neurodegeneration and acute nerve damage. J Clin Invest, 125 (4), 1433-1445. doi:10.1172/JCI79735 
Zhao, H., Lee, K. J., Daris, M., Lin, Y., Wolfe, T., Sheng, J., . . Meisen, W. H. (2020). Creation of a HighYield AAV Vector Production Platform in Suspension Cells Using a Design-of-Experiment Approach.Mol Ther Methods Clin Dev, 18 , 312-320. doi:10.1016/j.omtm.2020.06.004

\section{FIGURE LEGENDS}

Figure 1. Production of rAAV2-GFP by plasmid transfection

HEK-293SF cells in serum-free suspension were transfected with equimolar amounts ofpAdDeltaF6, pAAV$R C 2$, and $p A A V-C M V$-GFP to produce AAV2 vectors carrying a GFP transgene. a) Cell density in transfected and mock transfected cells tracked until harvest at $48 \mathrm{hpt}$. b) Volumetric vector yield in terms of nuclease-resistant vector genomes measured by digital droplet PCR. Error bars represent SEM for n=4 biological replicates.

Figure 2. Comparison of transfection efficiency and assembled AAV capsid production in transfected cells producing AAV2-GFP vectors

HEK-293SF cells producing AAV2-GFP vectors by transfection were fixed 48 hpt and stained for assembled $A A V$ capsids. a) Gating for transfection marker (GFP) and assembled AAV capsids following exclusion of debris and singlet gating (not shown). Also shown are mock transfected negative controls and a positive control for capsid assembly consisting of cells infected with replication competent (RC) AAV2 with a hAd5 helper virus b) Quantification of flow cytometry results from $n=3$ biological replicates. Error bars represent SEM.

Figure 3. Expression of transfected plasmids during AAV2-GFP vector production

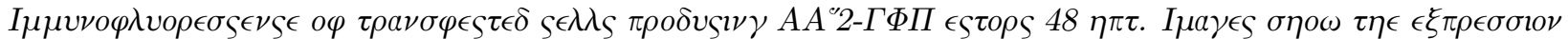

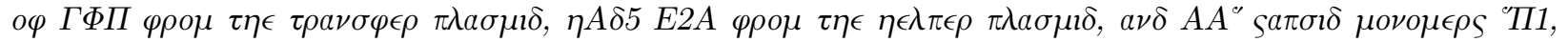

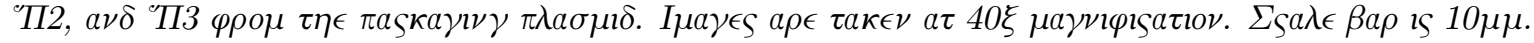


a

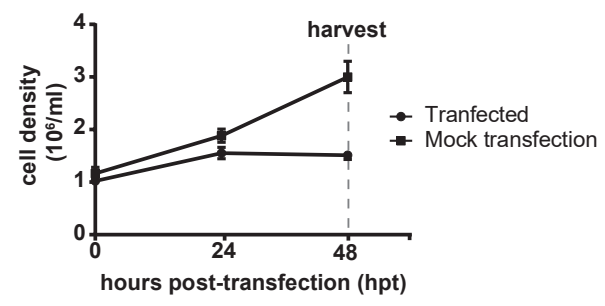

b

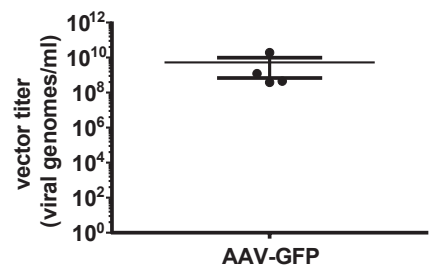


a

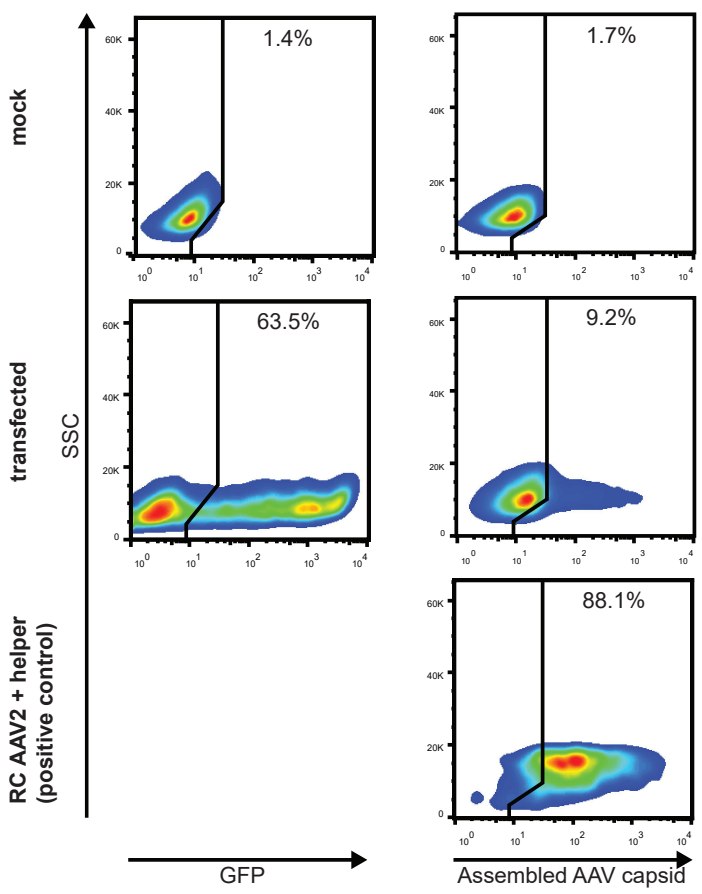

b

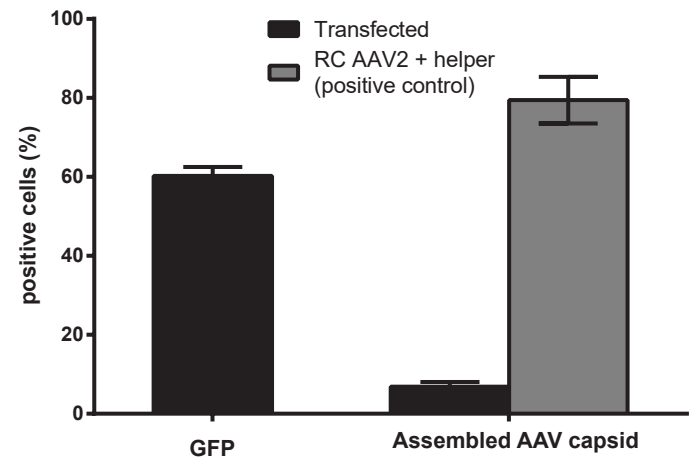

\section{Hosted file}

Figure 3.pdf available at https://authorea.com/users/446019/articles/545369-only-a-smallfraction-of-cells-produce-assembled-capsids-during-transfection-based-manufacturing-ofadeno-associated-virus-vectors 